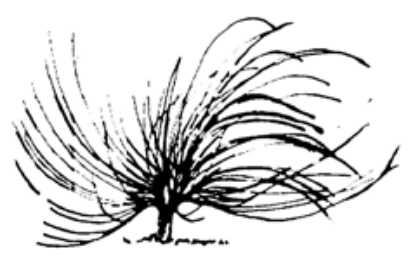

\title{
Ubicuidad y movilidad de herramientas virtuales abren nuevas expectativas formativas para el estudiantado universitario
}

\author{
María Luisa Sevillano-García \\ Universidad Nacional de Educación \\ a Distancia Madrid, España \\ mlsevillano@edu.uned.es \\ Esteban Vázquez-Cano ${ }^{3}$ \\ Universidad Nacional de Educación \\ a Distancia Madrid, España \\ evazquez@edu.uned.es
}

\author{
María del Pilar González-Flores ${ }^{2}$ \\ Universidad Veracruzana \\ Veracruz, México \\ pgonzalez@uv.mx \\ Luis Rey Yedra ${ }^{4}$ \\ Universidad Veracruzana \\ Veracruz, México \\ lyedra@uv.mx
}

\begin{abstract}
Resumen
El aprendizaje móvil es un campo de investigación y práctica educativa en rápida expansión, por lo que se hace necesario conocer su potencial en la educación y el aprendizaje; en este trabajo se presentan resultados de un estudio con los objetivos de conocer, identificar y valorar
\end{abstract}

\section{(ब) (1)}

Recibido: 12 de abril de 2016-Aprobado: 22 de setiembre de 2016

1. Catedrática de Didáctica y Nuevas Tecnologías aplicadas a la Educación de la Universidad Nacional de Educación a Distancia. Doctora en Filosofía y Ciencias de la Educación por la UNED.

2 Profesora-investigadora del Instituto de Psicología y Educación de la Universidad Veracruzana. Doctora en Educación por la Universidad Nacional de Educación a Distancia.

3 Profesor asociado del Departamento de Didáctica Organización Escolar y Didácticas Especiales de la Facultad de Educación de la Universidad Nacional de Educación a Distancia. Doctor en Ciencias de la Educación UNED.

4 Profesor-investigador de la Facultad de Pedagogía y del Instituto de Psicología y Educación de la Universidad Veracruzana. Doctor en Orientación y Desarrollo Humano por la Universidad Iberoamericana-Ciudad de México. CE: 
usos, frecuencia, beneficios, dificultades que representan los dispositivos móviles para posibilitar los aprendizajes ubicuos y móviles del estudiantado universitario. Por medio de cuestionarios y protocolos de historias de vida se accedió a una muestra intercontinental en la que participaron 875 estudiantes de 8 países. Los principales hallazgos muestran que, sobre el teléfono móvil, el ordenador portátil sigue teniendo preferencia para el acceso a internet, así como para realizar actividades académicas: elaboración de trabajos, el estudio, búsqueda de información, intercambio de apuntes y trabajos grupales. Sin embargo, el teléfono móvil va aumentando aceptación entre estudiantes para lograr el aprendizaje.

Palabras clave: Educación superior, formación en línea, ambientes virtuales de aprendizaje, aprendizaje virtual, estudiantado universitario, aprendizaje ubicuo.

\begin{abstract}
Mobile learning is a field of research and educational practice that is rapidly expanding, so it is necessary to know its potential in education and learning. In this paper, we report on the results of a study with the objectives of knowing, identifying, and assessing applications, frequency, benefits, difficulties that mobile devices represent to enable ubiquitous and mobile learning of college students. Through questionnaires and protocols of life histories, an intercontinental sample with the participation of 875 students from eight countries was accessed. The main findings show that the laptop still is preferred for Internet access over the mobile phone, as well as for academic activities: preparation of work, study, search for information, exchange of notes and group work. However, the mobile phone is increasing its acceptance among students to achieve learning.
\end{abstract}

Keywords: higher education, online training, virtual learning environments, virtual learning, university, ubiquitous learning 


\section{Fundamentación teórica. Una situación nueva en el aprendizaje universitario}

Las reformas en la universidad, generadas, provocadas y urgidas por la implantación de estudios siguiendo el denominado Plan Bolonia, han propiciado una situación nueva, hasta revolucionaria. En la actualidad, los dispositivos móviles han modificado el espacio fijo, lo cual conlleva a que el proceso formativo pueda efectuarse desde cualquier punto: ciudad, carretera, restaurante, ámbito local, internacional. Wifi gratis vemos anunciado en los quioscos, bares, hoteles. El 13 de enero de 2012, el diario español El Mundo publicaba en su sección Tribuna una reflexión titulada: Un modelo universitario para el siglo XXI. Entre otras observaciones se podía leer:

Tenemos que empezar a utilizar las plataformas tecnológicas que permitan que en tiempo real profesores y estudiantes del mundo entero interactúen, haciendo que la distancia y el tiempo no sean obstáculos y todos, con independencia de su horario laboral y su ubicación en el mundo reciban los nuevos conocimientos universitarios. Hemos de establecer plataformas que con el mínimo coste permitan que todo el caudal de sabiduría y buen hacer de nuestros mejores docentes se pueda comercializar de forma privada en el resto del mundo.

La tecnología ha revolucionado la vida del ser humano y está también presente en el ámbito educativo. La evolución de los dispositivos móviles ha sido veloz y universal, pero apenas ha permitido reflexionar sobre su integración sistemática y formativa en el ámbito educativo. Actualmente, estos recursos multiplican sus aplicaciones y abren múltiples posibilidades educativas, también favorecen acciones socializantes e inclusivas en personas con necesidades especiales. "La formación de la ciudadanía, escribimos en 2011 (Vázquez-Cano, Sevillano-García, Méndez-Pérez, 2011, p. 183), -requiere actualmente una atención específica en la adquisición de los conocimientos necesarios para tomar decisiones en el uso de objetos y procesos tecnológicos, resolver problemas relacionados con ellos" y utilizarlos para aumentar la capacidad de saber actuar y servirse de estos mismos en la búsqueda y consecución de un mejor aprendizaje. 
La rápida evolución de las tecnologías informáticas va pareja a la universalización de su uso, y es destacable el caso de los dispositivos móviles, que se incorporaron a la vida de las personas como una herramienta indispensable en toda actividad cotidiana. En México, la Asociación Mexicana de Internet (2015) reporta el uso de laptop (ordenador portátil) para conectarse a internet por un $68 \%$ de la población general y el uso de teléfono móvil (Smartphones) con el mismo fin por $58 \%$. El Centro de Estudios Especializados en Trastornos de la Ansiedad informaba (CEETA, 2015) que el uso de las diferentes aplicaciones consume hasta el $86 \%$ del tiempo de ocupación de la población. Del mismo modo, se puede ver que en España el 29\% de jóvenes entre 18 y 24 años aseguran no poder vivir sin el teléfono móvil (Diario ABC.es, 2015). Este hecho nos debe llevar, desde la educación, a buscar caminos, estrategias y contenidos para utilizar e integrar este dispositivo debidamente en la vida académica estudiantil. La complejidad, variedad y dinamismo evolutivo de estos equipos ha dificultado hasta la fecha un sosegado análisis de los efectos en las distintas áreas donde puede tener impacto su utilización. Es innegable su presencia en el ámbito educativo, aunque se hace preciso un análisis de su evolución en los últimos años y una descripción de las posibilidades tecnológicas y formativas que aportan el nuevo desarrollo del software y hardware portátil.

Creemos que los procesos actuales de transformación, en su esencia, se pueden caracterizar por un cambio hacia un nuevo paradigma de aprendizaje colaborativo, conectivo y heterárquico, lo que implica profundas modificaciones:

- $\quad$ Desde la perspectiva del estudianteado, los factores clave para la innovación de la educación serán la necesidad de competencias nuevas e implementar las necesidades de movilidad para una educación superior global y no delimitadas a unas fronteras.

- Desde la perspectiva del profesorado, tendrá lugar una redefinición del equilibrio entre enseñanza, aprendizaje e investigación hacia el desarrollo de la innovación y la competencia. El aprendizaje se reorientará a lo largo de paradigmas de colaboración, reflexión e interacción.

Especialistas y profesionales de la academia creen que la innovación permanente es el paradigma que coherentemente caracteriza los 
cambios necesarios en el panorama de la educación superior, como reflexiona Trillo (2015); por su parte, Quicios (2015) describe las formas en que esta transformación es demandada por el estudiantado y que sus usos formativos no están tan generalizados como se quiere hacer creer, en parte debido a la resistencia al cambio instructivo. Asimismo, no se debe dejar de lado la revolución que ha significado para la vida del ser humano el avance de la tecnología, particularmente en el ámbito educativo en el que abren múltiples posibilidades (Sevillano, 2015). Hay que preparar al alumnado para un futuro digital que ya es presente. La tecnología nos permite estar en diferentes lugares al mismo tiempo y el concepto de ubicuidad trae aparejada una profunda convergencia tecnológica entre todos los medios y la coexistencia de lo real con lo virtual, además de disponibilidad de la información a cualquier hora, desde cualquier parte y con cualquier dispositivo. Este tipo de interacción entre distintos dispositivos y medios sociales ha de modificar la manera como experimentamos el mundo y cómo enseñamos. Las dimensiones de un aprendizaje ubicuo parecen ser la continuidad en el tiempo y la interrelación contextual, que ayudan a cerrar brechas temporales y espaciales. Se constata una revolución en los procesos, los contenidos, los agentes, los recursos y los espacios en la enseñanza-aprendizaje. Entre los aspectos de este nuevo aprendizaje podríamos destacar la exploración, discusión, argumentación, colaboración y reflexión.

El aprendizaje ubicuo representa un nuevo paradigma educativo que en buena parte es posible gracias a los nuevos medios digitales. La convergencia de tecnologías y la proliferación de nuevos servicios basados en audio y video permiten que la educación actual esté disponible en todo momento, en cualquier lugar, en cualquier medio social y, lo más importante, usando cualquier dispositivo. En los planteamientos del aprendizaje ubicuo con herramientas móviles se ha de considerar el trinomio información, comunicación y conocimiento. $\mathrm{Y}$ en torno a estos tres ejes, se articularían las siguientes cinco macrocompetencias básicas: buscar, comunicar, organizar, producir, publicar.

Si bien podemos constatar que las nuevas generaciones crecen con una cierta y nueva sensibilidad y habilidades, esto les lleva, con frecuencia, a infrautilizar herramientas virtuales y a desencadenar procesos de abandono o pasividad. Personas adultas y mayores, por otra parte, encuentran resistencia inicial y, más que profundizar en las posibilidades manifiestas y latentes de estas herramientas, adquieren 
ciertas destrezas, más bien mecánicas que creativas e investigadoras. Como consecuencia, con cierta frecuencia, nos encontramos ante grandes inversiones en tecnologías de la información y la comunicación en los centros de formación y poca utilización por carencia en el dominio de estrategias pertinentes.

Las personas mayores, en el uso del tiempo libre (como posibilidad de entretenimiento, culturización y también transferencia del conocimiento), podrían encontrar en estas herramientas un cauce de autosatisfacción y participación madura en la conformación de la sociedad del conocimiento. Todos los sectores de la sociedad, jóvenes y adultos en busca de un empleo, en la administración, en el hogar, en la participación ciudadana, en su formación permanente y a distancia, en su experimentación de vida total, pueden verse muy beneficiados por estas herramientas que posibilitan un aprendizaje móvil y ubicuo.

Enseñar y aprender en las situaciones creadas por las nuevas tecnologías exige flexibilidad, espacio y tiempo personal y en grupo, menos contenidos fijos y procesos abiertos de investigación y comunicación. La construcción de conocimiento, a partir de las tecnologías impregna a las personas de una mayor motivación e interés hacia el aprendizaje, además de facilitar la interacción entre estudiante-docente, docente-estudiante, así como del alumnado entre sí y docentes entre sí. Asimismo, posibilita el desarrollo de la autonomía en el proceso de aprendizaje y su autorregulación.

La herramienta ubicua está concebida como un instrumento complementario en la formación, permitiendo al personal docente ofrecer contenidos formativos a sus estudiantes en diferentes horarios, facilitando el desarrollo de actividades educativas recomendadas para horario extracurricular. Su principal aliciente es lograr un ambiente educativo ágil, que pueda adaptarse a los cambios que la sociedad moderna exige, con actores -directivos, docentes y estudiantes- que participen activamente en comunidades virtuales educativas, donde se desarrollen prácticas en forma colaborativa, con mejor y mayor interacción con el contexto sociocultural, aligerando la fluidez del manejo informacional con otras instituciones y los participantes que en ellas se encuentran.

La educación ubicua se centra en cómo sacar provecho de la enorme cantidad de información al alcance de todas las personas y la posibilidad de disponer de esta misma en cualquier momento y lugar. Lo más grandioso de este fenómeno es la posibilidad de aprender en 
cualquier situación o contexto, aprender en, con, de y desde el entorno en sentido restringido y amplio. Algunos estudios sostienen que las herramientas virtuales posibilitan que prácticamente cualquier persona puede producir y diseminar información, de modo que el aprendizaje puede tener lugar en cualquier momento y en cualquier lugar.

A partir del aprendizaje m-learning o móvil, y la posibilidad de aprender a través de dispositivos móviles, las instituciones de formación tienen que explorar nuevas metodologías de enseñanza. Desde esta nueva realidad es necesario asumir la transformación de la educación, concibiendo al alumnado como participante creativo y comunicativo del proceso de aprendizaje. El personal docente puede crear aulas virtuales que se correspondan con las asignaturas que tengan a su cargo. La eficacia de los entornos educativos ubicuos descansa en la posibilidad de integrar, dentro de un mismo diseño instruccional, una serie de principios y bases pedagógicas adecuadas a las metas de aprendizaje, junto con las tecnologías de mediación que cumplan con esas bases y todo ello en el marco de un escenario de práctica donde puedan ensamblarse los elementos anteriores. Para que los aprendizajes en situaciones de ubicuidad sean efectivos, el uso de los dispositivos de mediación ha de responder a metodologías adecuadas y apoyarse en las herramientas necesarias que propicien la adquisición de conocimientos. Esto sitúa el centro de interés en el diseño de escenarios socio-técnicos que den cabida a experiencias prácticas y situaciones donde el aprendizaje se desarrolle de un modo fluido.

El aprendizaje ubicuo hace referencia al desarrollado en cualquier momento o lugar a través de dispositivos móviles. Este nuevo concepto abre un espectro de posibilidades técnicas y conceptuales para integrar las nuevas tecnologías en la enseñanza. El significado de la palabra ubicuo es sinónimo de omnipresente, estar en todas partes. En cualquier lugar del mundo se puede acceder/obtener y difundir información gracias a la evolución tecnológica, así como a la generación móvil, sin olvidar la capacidad de evolución humana.

La introducción del concepto de ubicuidad deriva de la computación ubicua, la cual se entiende como la integración de la informática en el entorno del individuo, de forma que los dispositivos no se perciban como objetos aislados. Debido a que la tecnología ha experimentado un gran avance en los últimos años, podemos disponer de una gran cantidad de información en cualquier parte y en cualquier momento. El 
extraordinario desarrollo de los dispositivos digitales, en los últimos tiempos, hace que el aprendizaje ubicuo no sea ya tan solo una posibilidad práctica, sino un imperativo social (Cope y Kalantzis, 2009).

El aprendizaje móvil es un campo de investigación y práctica educativa en rápida expansión. Sin embargo, existe todavía poco trabajo teórico y conceptual con el que explicar la compleja relación entre las características de la evolución tecnológica rápida y, a veces revolucionaria, su potencial de educación y aprendizaje, así como su integración en la vida cotidiana de los usuarios y las usuarias. La dimensión antropológica en la correlación de aprendizajes entre oportunidades, deseos y capacidades se encuentra en el desarrollo de la competencia para la autoorganización y autonomía del proceso de aprendizaje, lo que implica, cuando se trata del aprendizaje ubicuo, una formación y capacitación en orden a concienciar al estudiantado sobre las posibilidades de los dispositivos móviles y la elección de contenidos y estrategias más pertinentes. El aprendizaje mediante dispositivos móviles se rige por una relación triangular entre las prácticas culturales, las estructuras sociales y la acción de estudiantes en el proceso educativo.

\section{Diseño científico del estudio}

\section{Objetivos}

1. Valorar los usos, frecuencia, beneficios, dificultades que los dispositivos ordenador portátil y teléfono móvil en sus dimensiones de posibilitadores de aprendizajes universitarios ubicuos y móviles representan para el estudiantado actual.

2. Identificar tendencias actuales en jóvenes estudiantes de universidad a utilizar el teléfono móvil y el ordenador portátil en relación con su formación.

3. Verificar habilidades y competencias que estiman necesarias y que practican en el uso de las herramientas.

\section{Muestras}

Nos servimos de una muestra intercontinental de 875 estudiantes provenientes de diversas universidades de España (Complutense de Madrid, Vigo, Oviedo, Granada y Universidad Nacional de Educación 
a Distancia), Alemania (Otto-Friedrich-Universität Bamberg Bamberg), Italia (Freie Universität Bozen Brixen), Chile (Universidad del Libertador Bernardo O’higgins), Perú (Universidad Nacional Hermilio Valdizán-Huánuco), Colombia (Universidad de Cartagena, Fundación Universitaria Tecnológico de Comfenalco de Cartagena), Panamá (Universidad Pública de Panamá) y México (Universidad Veracruzana-Xalapa), distribuidos de la forma siguiente (tabla 1):

\section{Tabla 1}

Distribución de la muestra intercontinental

\begin{tabular}{ll}
\hline País & Número de estudiantes \\
\hline España & 451 \\
Alemania & 19 \\
Italia & 21 \\
Chile & 98 \\
Perú & 42 \\
Colombia & 110 \\
Panamá & 29 \\
México & 105 \\
\hline Total & 875 \\
\hline
\end{tabular}

Nota: Elaboración propia (2015).

\section{Instrumentos}

Cuestionario. Elaborado y validado por el sistema de personas expertas se utilizó un cuestionario con preguntas cerradas y abiertas cuya fiabilidad, según Alfa de Cronbach, es de .920 y fue aplicado por el equipo que presenta esta investigaciónen en las diversas universidades. Para este estudio hemos seleccionado algunas de las preguntas relacionadas y pertinentes con los objetivos pretendidos.

De las opciones de respuesta Nada, casi nada, de vez en cuando, a menudo y siempre, para el análisis de los resultados en este artículo 
nos hemos inclinado por considerar las dos primeras como negativas conformando la categoría casi nada (o no lo usan) y las tres siguientes positivas que conforma la categoría a menudo (lo usan), esta última es la que estaremos registrando para la presentación de los datos.

Protocolos de historias de vida. Con el propósito de conocer las dinámicas socioculturales que soportan el uso de dispositivos móviles y conseguir algunos elementos dinámicos, vivenciales, existenciales e importantes para comprender el uso que se da por parte de los universitarios y las universitarias a los dispositivos analizados, se realizaron historias de vida que ofrecen formulaciones que, aunque no responden de forma literal a las concepciones teóricas, sí permiten conocer la realidad de sus competencias y habilidades en el manejo de estos.

A lo largo de la descripción de los resultados de este estudio se incluyen algunas unidades de registro para contextualizar las percepciones personales de los sujetos usuarios de los dispositivos digitales móviles, las cuales pueden revelar yuxtaposiciones de los diversos contextos a través de experiencias individuales, en un principio desconocidas.

\section{Resultados e interpretación}

\subsection{Cuestiones exploratorias}

Un interés inicial estriba en conocer los medios que utilizan el estudiantado universitario para realizar su conexión a internet, para lo cual se hizo una pregunta expresa.

En la tabla 2 se observa una tendencia homogénea en el estudiantado universitario de los diversos países para el uso del ordenador portátil. Panamá presenta el porcentaje más bajo para hacer su conexión a internet por este medio. En cambio, acceder a internet mediante el teléfono móvil presenta una distribución más variada: Panamá y Chile son los que muestran porcentajes más elevados. Así, en general, se observa que el ordenador portátil sigue siendo el dispositivo más usado para conectarse a la red.

Sin embargo, para el caso de México hay poca diferencia entre una forma de acceder y otra, esto resulta en un acceso superior a lo reportado por Carlos Ponce de la Asociación Mexicana de Internet, quien señala que para la población en general, en 2014, 49\% ingresó vía el teléfono móvil (Smartphone), para 2015, 58\% lo hizo así; lo que 
Tabla 2

Medios para conectarse a internet

\begin{tabular}{lll}
\hline & Ordenador portátil \% & Teléfono móvil \% \\
\hline España & 98.8 & 79.5 \\
Italia & 100 & 61.9 \\
Alemania & 100 & 84.2 \\
Chile & 93.4 & 91.2 \\
Perú & 87.8 & 59.0 \\
Colombia & 98.2 & 84.0 \\
Panamá & 84.6 & 92.9 \\
México & 89.5 & 85.7 \\
\hline
\end{tabular}

Nota: Elaboración propia (2015).

contrasta con el dato para estudiantado universitario de este estudio que corresponde a $85.7 \%$.

La conexión a internet me permite buscar todo tipo de fuentes para poder luego citarlas en mis artículos y solucionar todas las dudas que tengo (protocolo 8).

Tabla 3

El costo económico le dificulta o impide conectarse a internet

\begin{tabular}{lll}
\hline & Ordenador portátil \% & Teléfono móvil \% \\
\hline España & 6.2 & 67.0 \\
Italia & - & - \\
Alemania & - & 100 \\
Chile & 7.4 & 40.7 \\
Perú & 18.2 & 45.5 \\
Colombia & 5.7 & 43.4 \\
Panamá & 6.7 & 40.0 \\
México & 12.5 & 70.0
\end{tabular}


Los costos implicados para la conexión a través de dispositivos móviles como el teléfono pueden ser una dificultad para usar internet tal como se puede apreciar en la tabla 3, cuando se reportaron de este modo en países como Alemania, México y España. En cambio, para el uso del ordenador portátil el país que presenta esta situación, y en menor porcentaje, es Perú, en donde también hay alguna dificultad para hacerlo, tanto a través del móvil como del ordenador portátil.

\subsection{Utilidades académicas}

El uso de dispositivos móviles en las actividades académicas incluye principalmente la elaboración de trabajos, el estudio, búsqueda de información académica, intercambio de apuntes y la realización de trabajos con compañeros.

La tabla 4 muestra que el ordenador portátil ocupa puestos más altos en su empleo para elaborar trabajos académicos, y son Alemania, México y España los países con mayor puntuación, en contraste con el teléfono móvil que presenta valores menores, solo destaca Colombia que, cerca de la mitad de los sujetos encuestados (47.6\%) lo utiliza para realizar sus trabajos. Conviene resaltar la relación que se observa entre el uso menos frecuente del teléfono móvil para esta actividad y el costo que implica la conexión a internet; así, se observa que los países que presentaron los puestos más bajos en este aspecto son aquellos que señalaron que el costo de conexión era un impedimento para su uso frecuente.

En horario escolar utilizo el ordenador portátil para realizar las prácticas y ejercicios de las asignaturas (protocolo 341).

Colombia, Chile y Perú aparecen como los países en los que sus estudiantes utilizan más a menudo para estudiar el teléfono móvil, pero siempre con valores menores a los registrados en relación con el ordenador portátil, dispositivo empleado para el estudio con altos valores por todos los sujetos encuestados. Es significativo el caso de Alemania con el $100 \%$ de uso para el ordenador portátil.

Para llevar a cabo mi estudio empleo los apuntes y temario dado en clase y recogido en el ordenador portátil y a veces en el móvil, 


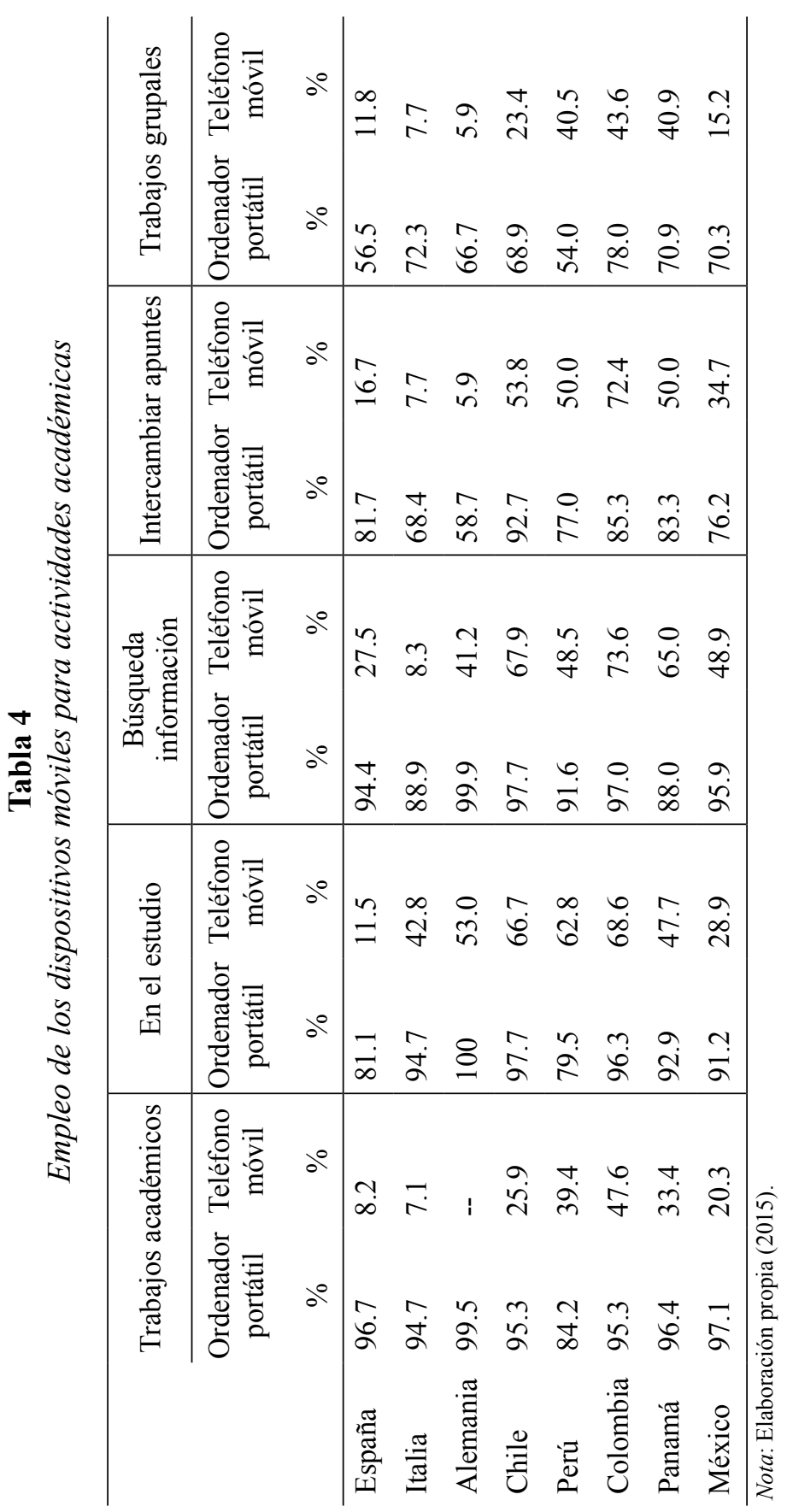


que suelo completar con información obtenida de internet para resolver mis dudas (protocolo 62).

El ordenador portátil lo utilizo en mi casa, para buscar la información que pueda necesitar para realizar los trabajos para mis clases, para pasar a limpio los apuntes que hasta principios de este año escribía a bolígrafo en clase (protocolo 199).

Conviene destacar que México y España son los países que arrojaron valores más bajos en el uso del teléfono móvil para el estudio.

Respecto de la búsqueda de información, el ordenador portátil sigue siendo la herramienta más utilizada; la información académica que implica becas, calificaciones, exámenes, apuntes, recibe altos porcentajes de usos con el ordenador portátil por parte de todos los grupos encuestados. Se incrementa el uso del teléfono móvil; sin embargo, Italia y España siguen teniendo porcentajes bajos (8.3\% y 27.5\% respectivamente); para México esto representa el 48.9\%.

A la hora de hacer trabajos, muchas veces he utilizado el móvil tanto para recopilar información de forma instantánea dada su velocidad e incluso para ayudarme de sus aplicaciones para hacerlos (protocolo 92).

Para el intercambio de apuntes y el trabajo grupal no se privilegia tanto el uso de los dispositivos móviles; sin embargo, se observa una marcada preferencia por el ordenador portátil en contraste con el teléfono móvil, a pesar de que en los últimos años está siendo incrementado su uso. Tal vez, la frecuente insistencia en la competitividad lleva a no intercambiar el producto académico.

El teléfono móvil lo utilizo para ver y enviar apuntes entre mis compañeros, profesores y yo. Lo utilizo para cualquier urgencia que tenga que ver con lo académico; de hecho lo tengo siempre conectado y aunque lo ponga en silencio estoy siempre a disposición (protocolo 100).

El trabajo colaborativo lleva inculcándose como una dimensión enriquecedora de la personalidad y los trabajos académicos en la formación desde hace algún tiempo; se observa, sin embargo, un uso 
menor de los dispositivos para esta tarea en contraste con el que se les da para otras actividades. El teléfono móvil encuentra menor aplicación en general; los países que más reportaron que no lo usan son Alemania (94.1\%), Italia (92.3\%) y España (88.2\%) lo que resulta interesante, ya que en estos países particularmente se hace hincapié en este tipo de actividades de colaboración.

Cuando llego a casa utilizo el ordenador portátil que es más cómodo que el móvil y puedo realizar con él trabajos y hacer una lectura más pausada de lo que tenga que buscar o leer (Protocolo 146).

Utilizo el teléfono móvil para concertar citas con mis compañeros de facultad a la hora de hacer trabajos, asi como para el intercambio de información de cualquier tipo (Protocolo 80).

El ordenador portátil, nos da independencia para poder movernos de un lado a otro nos permite coger apuntes en la universidad, hacer trabajos de grupo sin necesidad de estar en un recinto cerrado. Lo utilizo para leer la prensa, completar apuntes, estar informada, conseguir apuntes colgados en la página web de la universidad (campus virtual) (protocolo 3).

\subsection{Nuevos espacios para el aprendizaje}

Estudiamos en este apartado, en primer lugar, los espacios preferidos, vinculados a la institución académica para el estudio. Es importante resaltar que los dispositivos empleados permiten, sin necesidad de conexión, la movilidad.

Así, en la tabla 5 se aprecia que el teléfono móvil tiene mayor uso en los diversos espacios escolares, a diferencia del ordenador portátil, el cual llega a tener un uso más frecuente en las aulas y la biblioteca; en el caso de México, ambos dispositivos presentan valores más homogéneos que para los demás países.

La cafetería no es lugar de referencia con el ordenador portátil, salvo en el caso de Colombia, con $66.0 \%$ para el portátil y $83.9 \%$ con el teléfono móvil. Este dispositivo sí experimenta mayor utilización en todos los países, salvo en el caso de Italia, que representa solo al 25.1\%.

Al reportar el uso de los dispositivos en los pasillos de la facultad, los datos de la tabla 5 muestran que este no es el espacio donde 


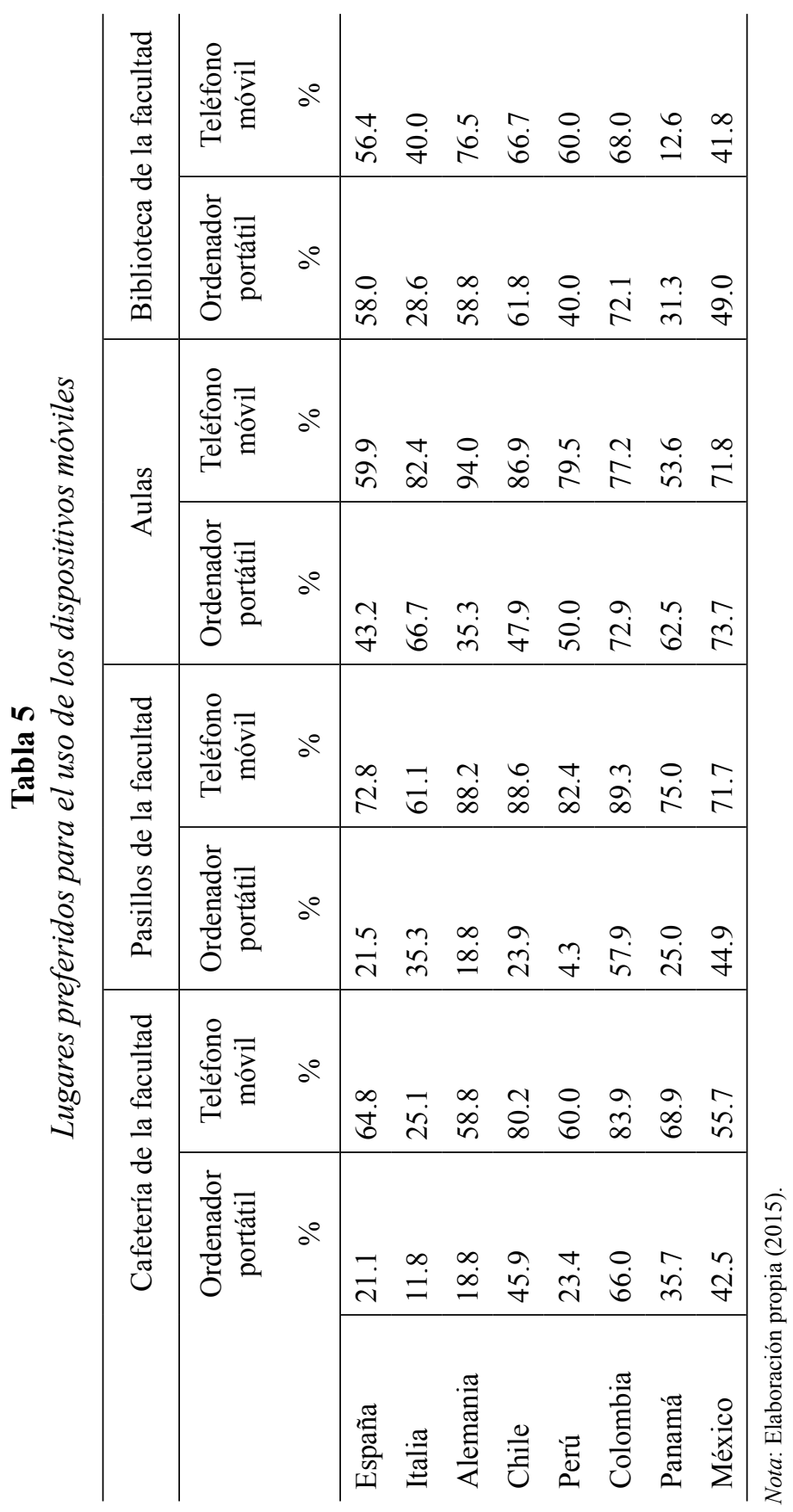


más se utiliza el ordenador portátil; sobresale el caso de Colombia con el nivel de preferencia más alto en ambos instrumentos $89.3 \%$ para el móvil y $57.9 \%$ para el ordenador. Obsérvese que en este caso el móvil se presenta con mayores puntajes en la categoría lo usa, tal vez debido a la facilidad de movilidad que representa.

El uso en las aulas es un tanto similar para el ordenador como para el teléfono. Presentan mayor diferencia en el uso de uno y otro Alemania con un $94.0 \%$ para el móvil en contraste con $35.3 \%$ para el ordenador y, Chile con $86.9 \%$ para el teléfono y $47.9 \%$ para el ordenador.

La biblioteca tampoco resulta ser el espacio de gran preferencia, salvo en el caso de Alemania con el $76.5 \%$ para el teléfono y Colombia con el $72.1 \%$ para el ordenador; para el resto de los países se presentaron porcentajes con pocas diferencias de preferencia entre uno y otro dispositivo.

Los lugares en los cuales utilizo los dispositivos móviles son variados y según las circunstancias, de esta forma el teléfono móvil lo uso en cualquier sitio que me encuentre siempre que las circunstancias me lo permitan, el ordenador lo utilizo fundamentalmente en casa, en la universidad o en cualquier lugar con zona wifi (protocolo 445).

Los tiempos pasados en espacios extraacadémicos como lugares de ocio, la casa o el medio de transporte son incorporados mediante los dispositivos móviles a la ampliación del tiempo y oportunidad de estudio (tabla 6). Globalmente el teléfono móvil es el preferido: Alemania $82.4 \%$, España $75.2 \%$ e Italia $73.3 \%$ destacan por aprovechar el tiempo que pasan en los transportes públicos con el teléfono móvil.

En este espacio el ordenador portátil registra pocos usos académicos. Cuando se refieren a las actividades en casa ambos instrumentos registran una alta utilización. En los lugares de ocio vuelve a ser el teléfono móvil el instrumento preferido: destaca Chile con un $86.6 \%$.

\subsection{Funciones atribuidas y usos practicados}

Es de nuestro interés conocer acerca de las funciones del teléfono móvil y del ordenador portátil que identifican así como del uso que les dan; se les proporcionaron varias alternativas de respuesta como: 


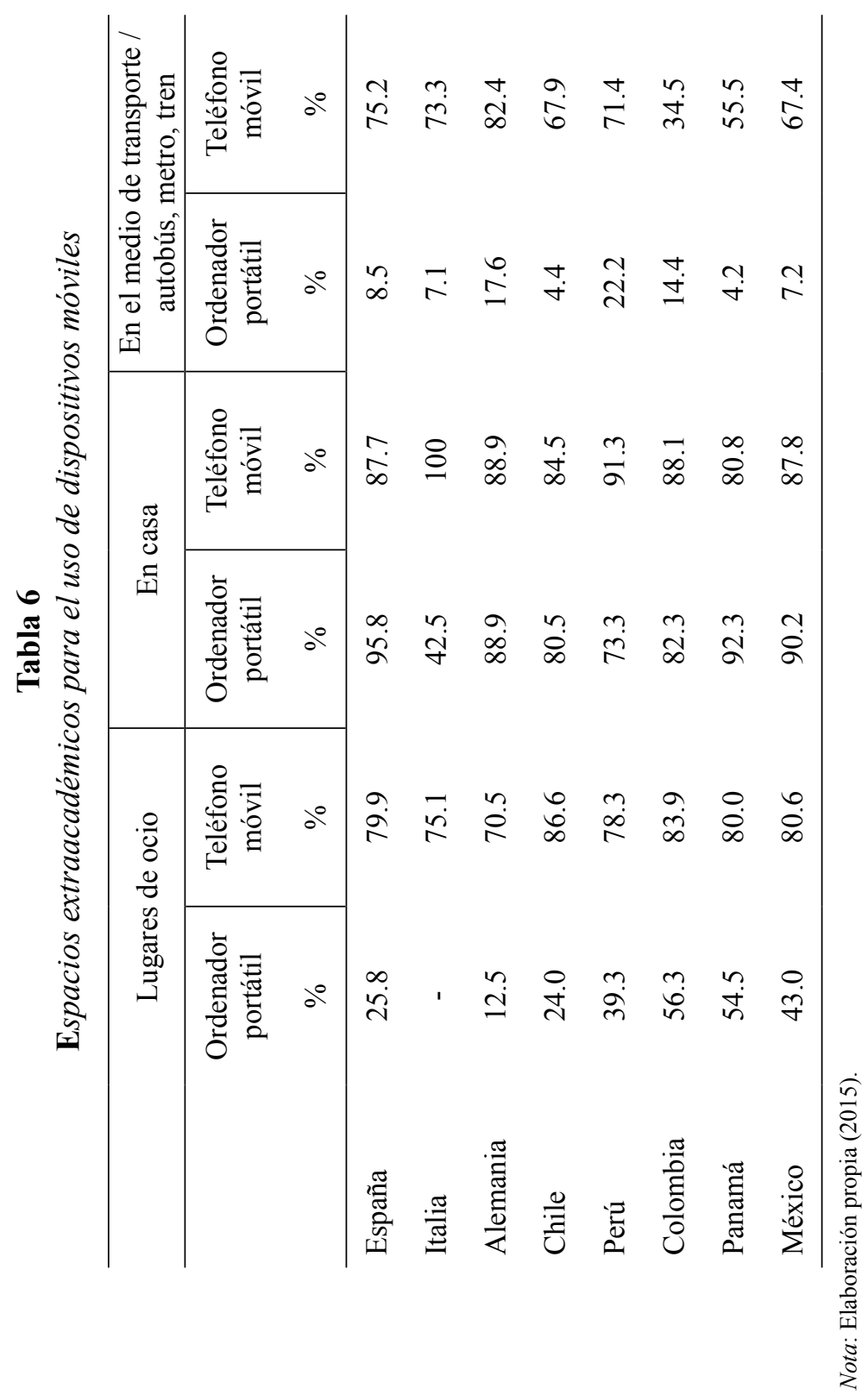


entretenimiento, expresiva, motivadora, informativa, instructiva, colaboradora, comunicativa, ilustrativa e innovadora.

Respecto de las funciones que identificaron en el móvil sobresalieron comunicativa y entretenimiento con 629 y 566 impactos absolutos respectivamente (de un total de 3,255); todos los países registraron en estas las puntuaciones más altas. En cambio, la función instructiva, con 155 impactos es la función menos valorada, lo que llama nuestra atención, dada la población a la que está dirigido el estudio (tabla 7).

Es observable que los sujetos participantes identifican más las funciones de comunicación al referirse al teléfono móvil y, al referirse al uso que le dan, sobresalen la comunicación vía mensajes o llamadas, escuchar música, uso para redes sociales y para jugar. En cambio, el uso para cuestiones más académicas no es muy identificado por los grupos participantes al haber recibido un bajo número de impactos $t a-$ reas de tipo académico (182) y todavía en menor cantidad organizar información (114). Debe destacarse que su uso es más reconocido que sus funciones al haber obtenido más impactos absolutos $(4,400)$ como se observa en la tabla 8 en comparación con las funciones atribuidas $(3,255$; ver tabla 7$)$.

En cuanto al ordenador portátil, las funciones que sobresalieron por el número de impactos para todos los países fueron la informativa con 647 , instructiva con 535 y comunicativa con 531 de un total de 4,121 impactos como se aprecia en la tabla 9. En México, también destacan la función colaboradora como segunda elección.

Al hacer referencia a la preferencia de uso que dan al ordenador portátil, se observa en la tabla 10 que los impactos totales obtenidos globalmente $(6,784)$ son muy superiores que para el caso del teléfono móvil y destacan usos más académicos. Así, se destacan: buscar información (656), recibir y contestar e-mail (644), organizar información (631) y tareas de tipo académico (628) como las más identificadas. 


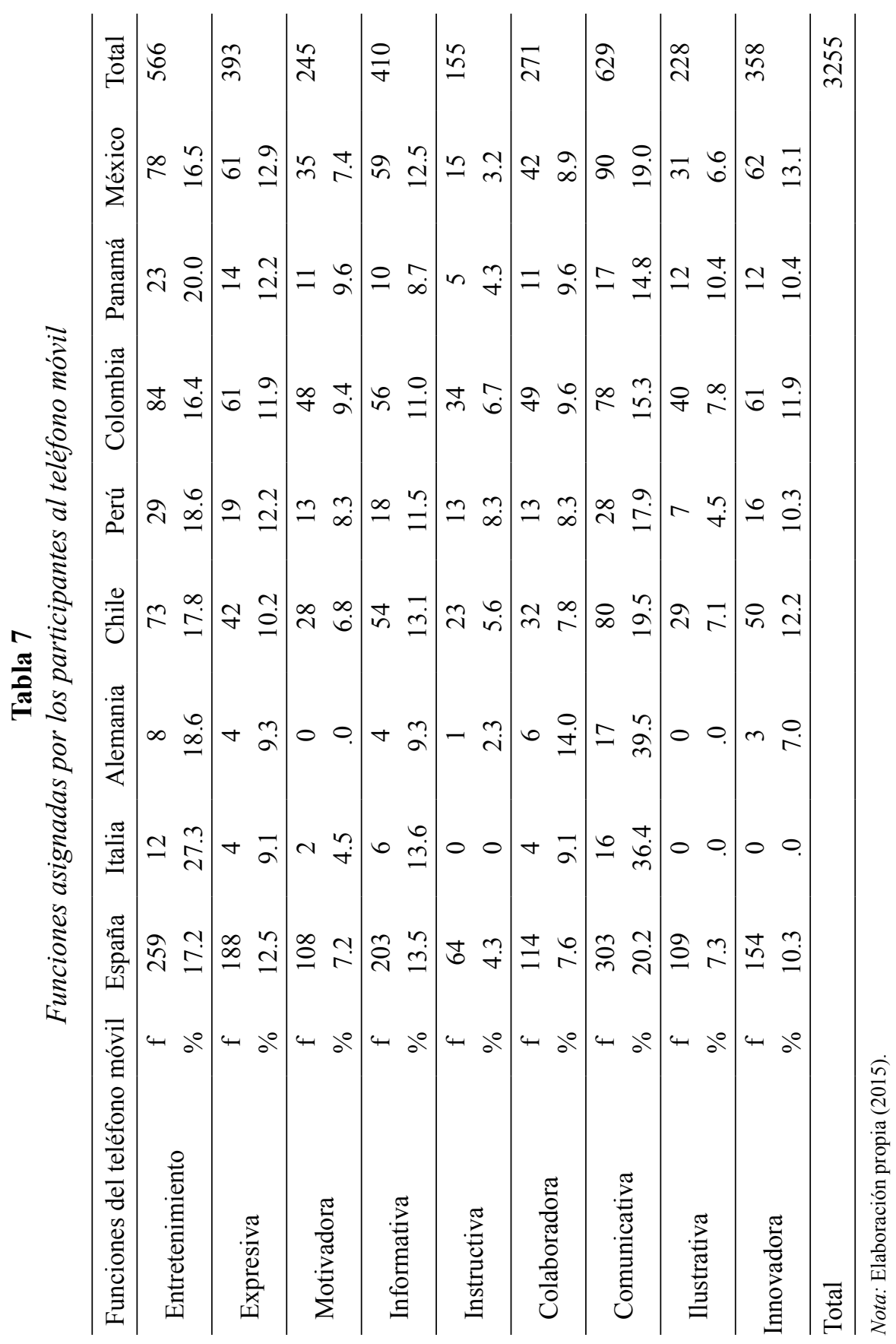




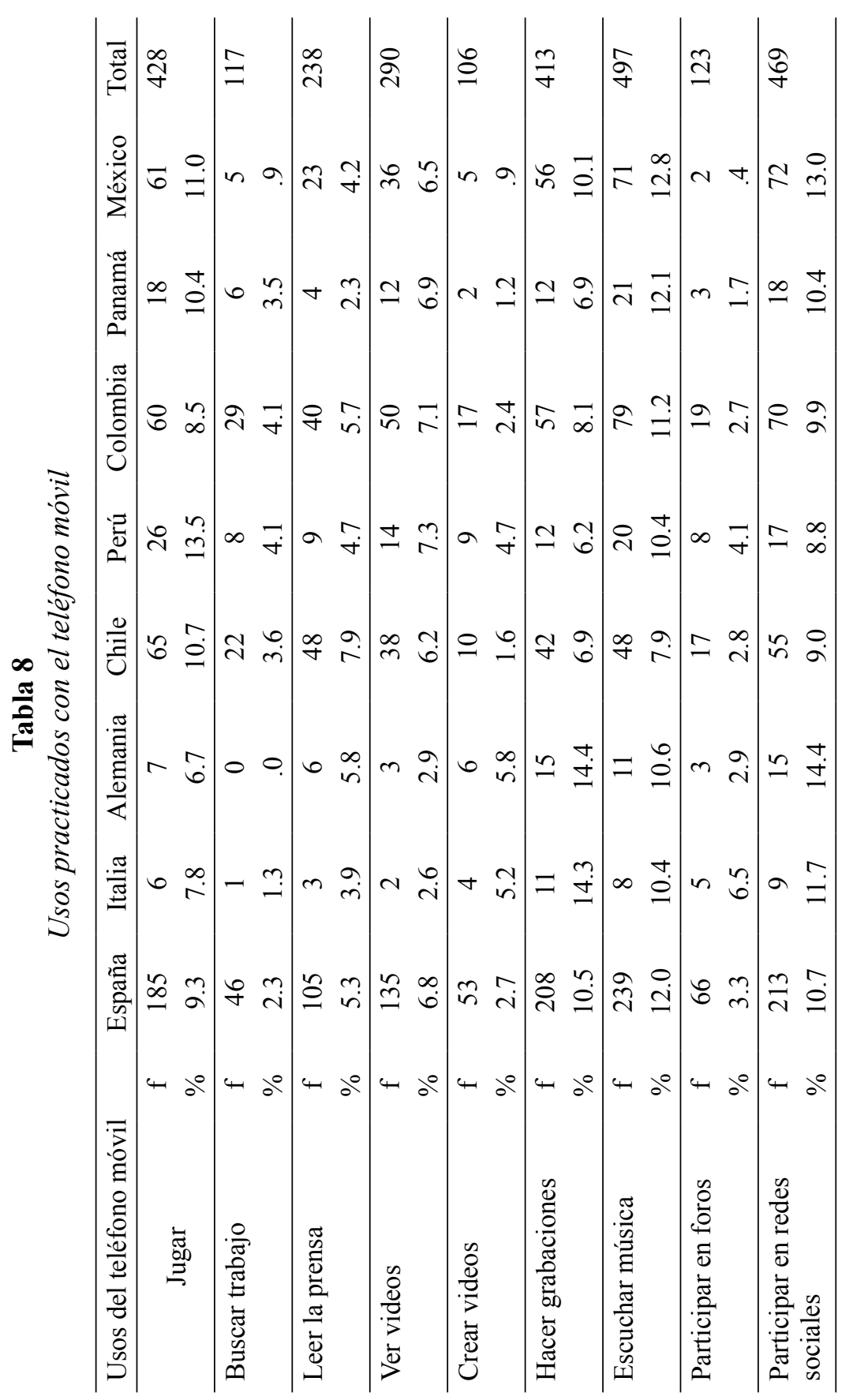




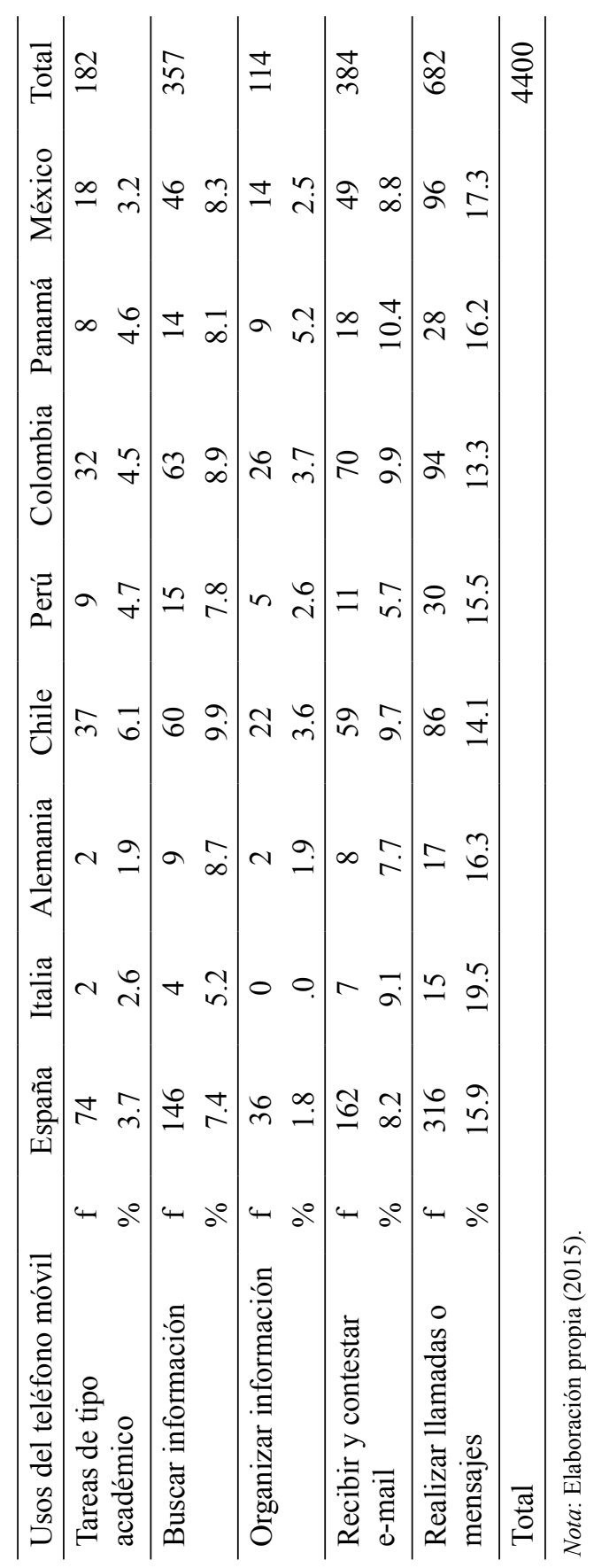




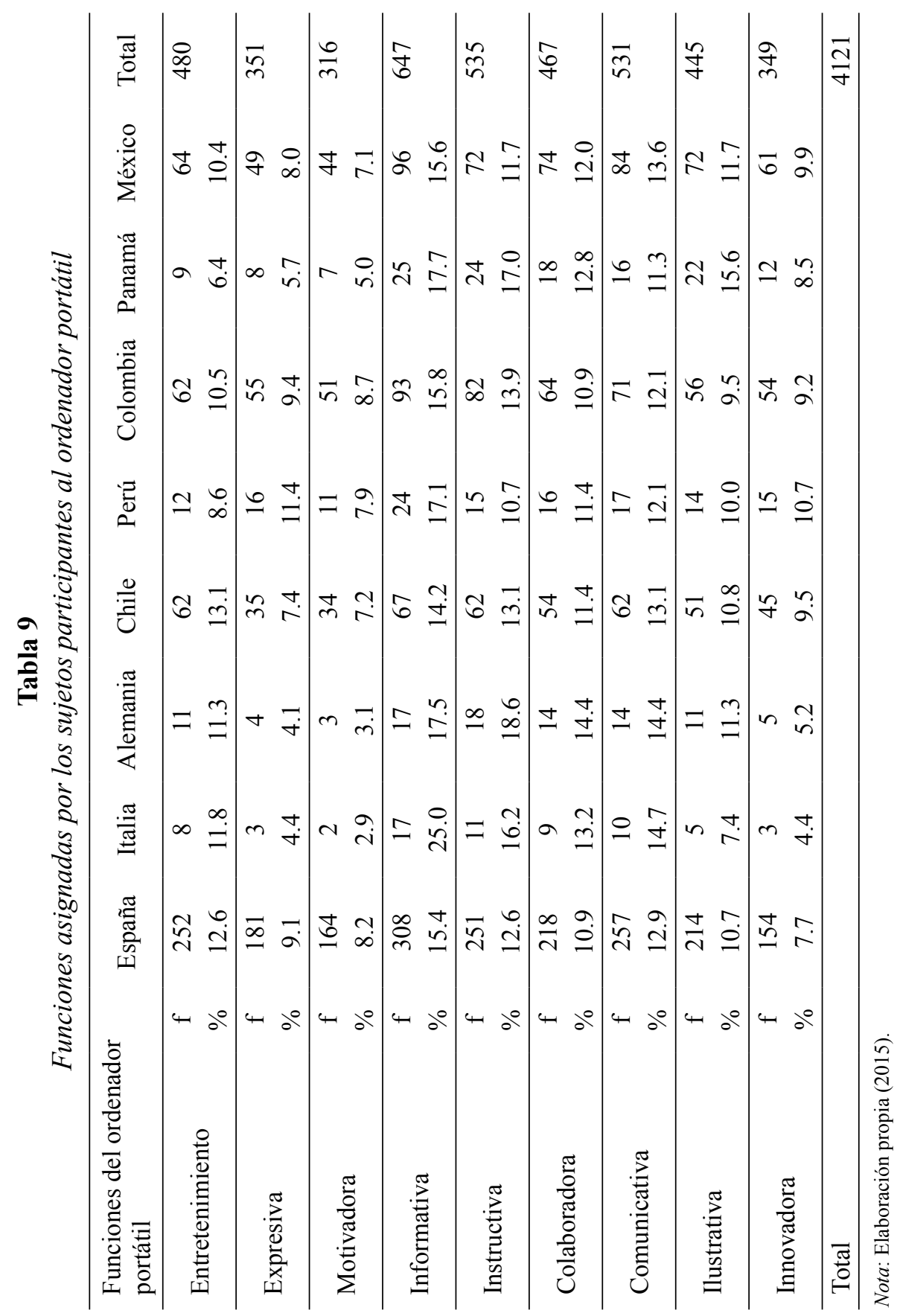




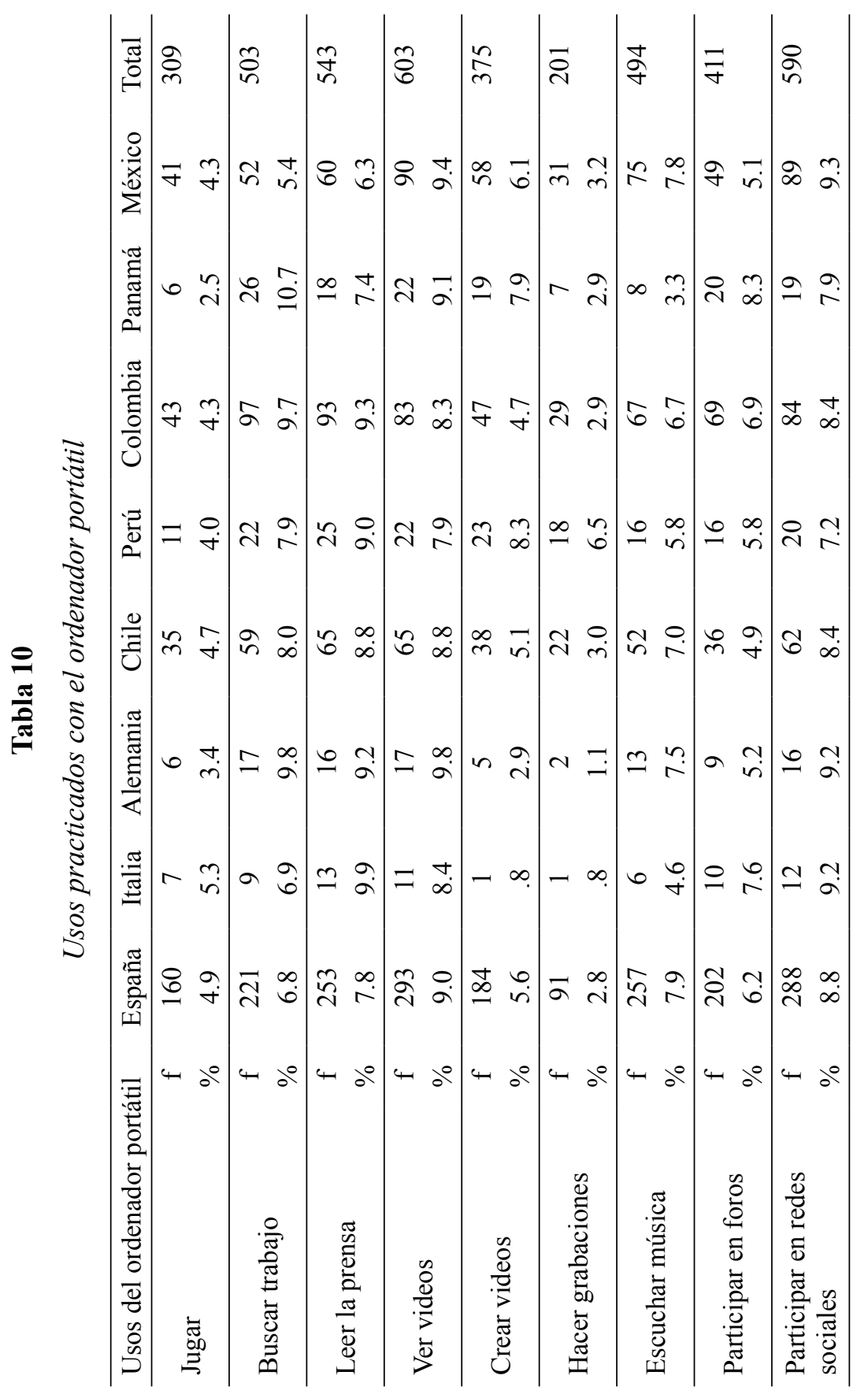




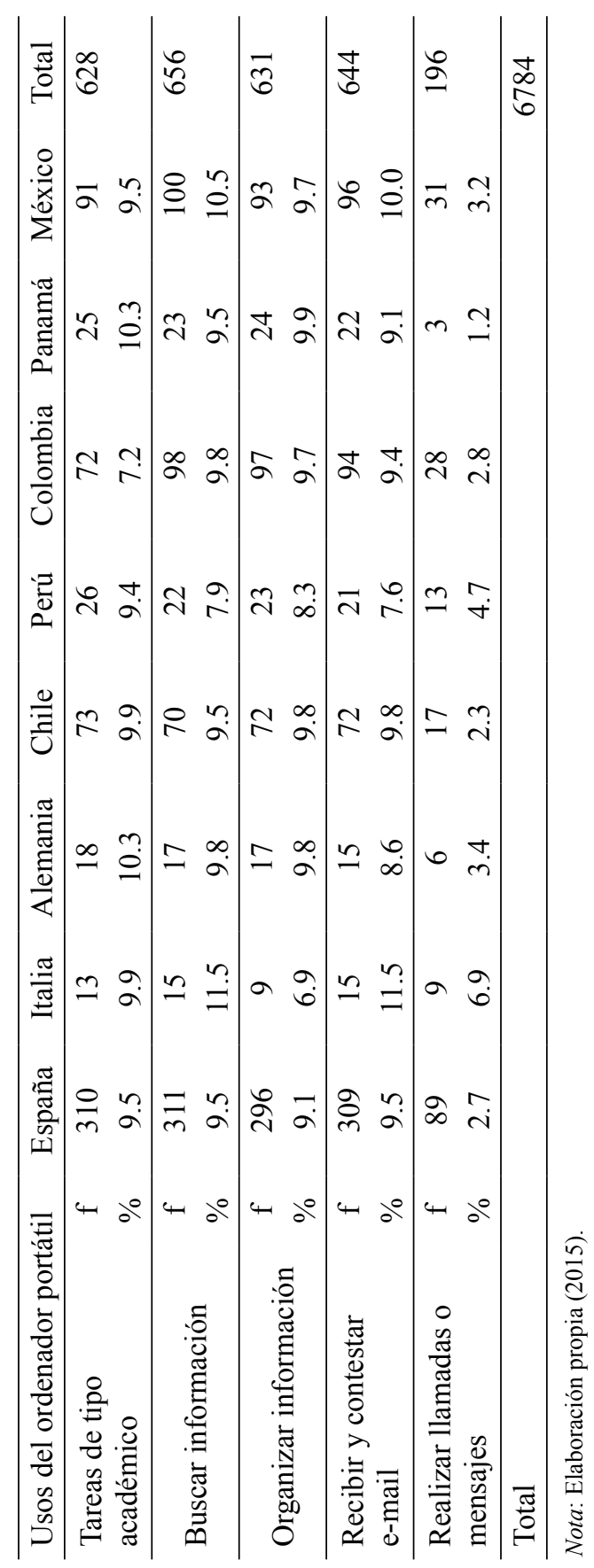


Respecto de las competencias necesarias para el uso de los dispositivos móviles, seis eran sobre las que tenían que discernir: Conocimiento del software; competencia digital; capacidad de adaptarse a las nuevas tecnologías; interés; manejo del paquete Acces, y capacidad para buscar información.

Para cada una de ellas, la primera opción se trataba a nivel conceptual de las que consideraban imprescindibles. En la segunda opción, aquellas que utilizaban.

Los resultados reflejan que la competencia más señalada (a nivel global 29.6\%) como imprescindible para manejar de forma adecuada el ordenador portátil es el conocimiento del software, donde es México el país que más lo mencionó (47\%); en cambio, Perú, lo indica solo el $5.0 \%$, pero reconoce una necesidad de competencia digital (35\%) este es el país que más la indicó, la cual obtuvo globalmente un valor de $20.3 \%$; España la señala $27.4 \%$ y México $14.5 \%$. El resto de las competencias obtuvieron porcentajes menores.

Se debería fomentar y facilitar el aprendizaje sobre ciertos programas de ordenador, de hecho creo que deberian tener los alumnos una asignatura donde aprender al menos lo básico para adquirir mayores destrezas y competencias (protocolo 100).

Asimismo, seis eran las competencias según su grado de utilización que tenían que jerarquizar para determinar el grado en que las utilizaban: Conocimiento del software; competencia digital; interés; capacidad para buscar y manejar información; navegar por internet; rapidez, y otras diversas. Esta opción se relaciona con la práctica.

Respecto del ordenador portátil, la competencia digital es globalmente la opción más destacada con un $18.5 \%$ en promedio del total de países, destaca México con el 35.9\%. El conocimiento del software correspondiente con un $17.8 \%$ es la segunda competencia más utilizada, seguida de la capacidad para buscar y manejar información (6.1\%), Chile es el país que más lo señala con $22.2 \%$ en ambas competencias. Todos los valores son menores que los indicados en el nivel conceptual.

Con el uso del ordenador portátil he aprendido mecanografia, escribir mejor y más rápido. He aprendido a utilizar programas que no sabía ni que existían como el Excel, Access y Publisher; a 
navegar rápida y eficazmente por Internet. Y algo que me gusta mucho sobretodo y de lo que he aprendido muchísimo son los programas de edición de música y vídeo, algo que hago en mis ratos libres. En general desarrollar competencias informáticas y ofimáticas que me sirven y ayudan en mi día a día para resolver problemas o hacerlo todo más fácil y rápido (protocolo 116).

Las competencias imprescindibles en el manejo del teléfono móvil sobre las que tenían que discernir eran: Conocer herramientas y funciones; capacidad de adaptación a las nuevas tecnologías; conocer terminología básica; saber realizar llamadas y recibirlas; saber enviar mensajes y otras diversas. En esta opción se trataba a nivel conceptual de aquellas que consideraban imprescindibles.

Para saber utilizar este tipo de teléfonos, es necesario tener cierto conocimiento de informática, ya que es un ordenador en miniatura (protocolo 144).

El comentario anterior se ve reforzado por los resultados obtenidos en el cuestionario que muestran que para el teléfono móvil las competencias que consideran imprescindibles son en mayor proporción el conocimiento de herramientas y sus funciones (32.0\%); México con el $46.2 \%$ y España con el $31.6 \%$ son los países que más la destacan. Saber realizar llamadas y recibirlas es el segundo lugar de opciones $(16.5 \%)$ Italia con un $50.0 \%$ es el país que más la destaca. Le siguen en el orden: Capacidad de adaptación a las nuevas tecnologías (4.5\%); saber enviar mensajes (2.4\%) y conocer terminología básica (1.9\%).

\subsection{Aprendizajes y beneficios buscados y encontrados}

Un punto de interés importante respecto del uso de los dispositivos móviles es el relacionado con los aprendizajes que se pueden buscar y lograr a través de ellos. Se trató de identificar, por una parte, las necesidades en cuanto al aprendizaje y, por otra, lo que sus usuarios y usuarias reportan haber encontrado por estos medios. Así, se quiere verificar si existe o no una consistencia entre lo demandado y lo que los dispositivos móviles pueden ofrecer. 
Los dos aprendizajes que buscan con el ordenador portátil y que fueron más señalados: Ayudas para realizar trabajos recomendados según programas de las disciplinas regladas y documentación para ampliar estudios o tareas son una preocupación de la mayoría de participantes en todos los países considerados, con un $44.2 \%$ y $36.0 \%$, respectivamente, en relación con el ordenador portátil.

$\mathrm{Al}$ contrastar estos datos con los obtenidos ante el cuestionamiento acerca de cuáles realmente consiguen, el 28.4\% y $41.3 \%$ manifiesta que sí encuentra esos aprendizajes. La existencia y hallazgo de documentación supera las expectativas de forma homogénea en todos los países. Destacan, sin embargo, Panamá con el $77.8 \%$ y Perú con el $71.4 \%$.

El uso de un ordenador portátil me ahorra muchísimo tiempo, puedo enviar trabajos desde cualquier parte y en cualquier momento, gestionar la información de que dispongo y buscar nueva sin necesidad de hacer malabares con un montón de folios y otros tantos libros; tengo la actualidad informativa al alcance de la mano en todo momento (Protocolo 201).

La información y la comunicación académicas buscadas por medio del teléfono móvil llega al $46.3 \%$ y la documentación al 9.0\%. La encontrada se mantiene un poco más baja, pero casi en los mismos niveles: la información y comunicación académicas encontradas llega al $41.7 \%$ y la documentación al $8.3 \%$.

Nos interesa conocer, también, el tipo de satisfacciones académicas que recibe el estudiantado en relación con los dos dispositivos analizados considerando: comodidad; rapidez; satisfacción académica y social; entretenimiento; ayuda académica, información e incremento de contactos sociales.

Sobre el ordenador portátil la satisfacción académica y social con un $26.6 \%$ de impactos promedio es la más alta para la totalidad de los países, son México (35.1\%) y España (34.5\%) los países más destacados. La información académica (becas, notas, conferencias, etc.) le sigue con un $16.8 \%$ promedio de aceptación, Colombia es el país donde más satisfacción se encuentra (27.3\%) seguido de Panamá (26.3\%).

Con respecto del teléfono móvil, el incremento de contactos académicos y sociales con el $25.1 \%$ promedio para la totalidad de los países es la más alta, seguida de la comodidad (22.7\%). Se debe destacar que su 
satisfacción relacionada con la ayuda académica es poco valorada (solo $1.3 \%$ en promedio), con los porcentajes más bajos conseguidos por España $(0.5 \%)$ y México $(2.9 \%)$; sin embargo, conviene aclarar que esta categoría no fue siquiera considerada por Italia, Colombia ni Panamá.

Los beneficios que manifiestan haber obtenido mediante el uso de estos dispositivos constituyen un crisol de resultados. De los cinco indicados (entretenimiento; ayuda en el aprendizaje; realización de trabajos; contactos sociales [chat, redes sociales] e información) para poder señalar los más valorados, se destaca que justo los dos menos académicos (el entretenimiento y los contactos sociales) fueron los menos estimados con una amplia diferencia del resto (3.0\% y 5.9\%) respectivamente. La información académica tanto institucional como de docentes, administración y compañeros y compañeras recibe, en el caso del ordenador portátil, una valoración global de $43.8 \%$, donde destacan Panamá con 56.5\%, Italia con 50.0\% y México con $46.9 \%$. La realización de trabajos académicos con 17.1\%, Panamá con el $30.4 \%$ ocupa un primer lugar en su aceptación, seguido de Colombia (23.0\%) y de México (20.8\%).

Para el teléfono móvil, los comportamientos son similares. Entretenimiento es la variable peor valorada con $5.2 \%$ de los impactos globales, seguida de acceso a internet con $6.5 \%$. Las mejor valoradas son accesibilidad a las personas con $41.6 \%$ de forma global, en donde se destacan México (66.7\%), Panamá (64.0\%) e Italia (60.0\%).

Es importante también conocer el grado de utilización de estos dispositivos para el aprendizaje universitario en donde se encontraron resultados interesantes en el sentido de que para el estudiantado universitario de algunos países como Italia (para ambos dispositivos) y Alemania (el teléfono móvil) estos dispositivos no se destacan como preferentes para actividades de aprendizaje (tabla 11). En cambio, para los otros países, el ordenador portátil es altamente valorado como forma de aprendizaje. Lo mismo ocurre con el teléfono móvil, aunque en un menor porcentaje; sin embargo, se deja ver que en algunos países como Panamá, Chile, Colombia y México se va posicionando como un dispositivo útil para lograr el aprendizaje. 
Tabla 11

Grado de utilización para el aprendizaje universitario

\begin{tabular}{ll|l}
\hline & Ordenador portátil \% & Teléfono móvil \% \\
\hline España & 96.8 & 52.5 \\
Italia & - & - \\
Alemania & 50.0 & 6.3 \\
Chile & 94.1 & 80.3 \\
Perú & 70.0 & 63.1 \\
Colombia & 96.0 & 80.0 \\
Panamá & 96.4 & 82.2 \\
México & 96.1 & 66.9 \\
\hline
\end{tabular}

Nota: Elaboración propia (2015).

\section{Discusión y conclusiones}

La investigación planteada, realizada y en parte preparada para esta revista representa una innovación de interés social, familiar, académico, metodológico y didáctico. Los resultados muestran que, en todos los países estudiados, las tendencias son más o menos homogéneas con algunas variantes. Estas están relacionadas con la situación económica, como es el caso de Perú, que a veces convierte en inviable la conexión al dispositivo móvil por los altos costes en relación con el índice de vida. En otros casos, el tiempo prolongado que emplean en el transporte público y como ocurre en España, singularmente en Madrid, es aprovechado para centrarse especialmente con el teléfono móvil y realizar tareas académicas. El caso de Colombia es paradigmático en relación con los pasillos de la Facultad, espacio preferido por sus estudiantes para conectarse y resolver singularmente vía ordenador portátil, tareas de estudio.

Existe, se constata empíricamente, una dualidad entre las competencias que manifiestan ser necesarias y las que luego emplean. Nos encontramos ante generaciones nativas digitales que entienden el manejo de estos dispositivos como algo intuitivo y que, además, con el tiempo y el mucho ejercicio llegan a adquirir.

Los resultados representan, a nuestro entender, una triple urgencia y demanda. La primera afectaría a las entidades propietarias de las 
telecomunicaciones para que, en aras de ir aminorando la brecha digital y favorecer los usos académicos e instruccionales de las modernas tecnologías móviles y ubicuas, ofrezcan tarifas más reducidas a estudiantes (como segmento débil en capacidad adquisitiva, principalmente en aquellos países en los que se han convertido en las casi únicas herramientas para acceder al conocimiento), ya que los libros impresos son pocos, costosos económicamente y no fáciles de adquirir comercialmente. Se ha dado el salto al universo digital, pero su acceso sigue siendo limitado por los altos costes de conexión, uso y mantenimiento.

La segunda instancia estaría en el profesorado universitario. Los usos frecuentes y crecientes, por parte de estudiantes, piden que sus docentes contemplen más contenidos curriculares en las redes y no solo y especialmente informaciones, aunque sean académicas. Si bien el intercambio de apuntes o trabajos se acredita como frecuente y útil, es menester propiciar una mayor información docente sobre los recursos digitalizados ya existentes en todas las áreas de conocimiento, para que el estudiantado, desde sus móviles, accedan a estos. En esta situación digital no podemos tener esos inmensos repositorios de recursos fosilizados por falta de conocimiento, tiempo o carencia de preparación o interés profesoral. El propio consorcio Erasmus, por ejemplo, está diseñando un gran portal multilingüe entre las más de 4000 instituciones que lo integran para la difusión de cursos masivos con el objetivo de interconectar conocimiento, investigación y transferencia de resultados entre las universidades y donde el formato audiovisual ubicuo y móvil será uno de los prioritarios (Vázquez Cano, 2013, p. 90).

La tercera instancia a la que nos llevan los resultados de este estudio es a mirar al propio estudiantado. De forma análoga a como durante mucho tiempo se cultivó una actitud y práctica analítica y crítica en relación con los mensajes y contenidos de toda naturaleza mediados por los medios impresos y audiovisuales, periódicos o no, cultivando una metodología específica, ahora entendemos que es menester diseñar un sistema de análisis tanto para la producción, como la explotación de los recursos a los que se puede acceder a través de los dispositivos móviles, como el teléfono y el ordenador portátil, cada vez más presentes en la sociedad mundial y en la vida académica de estudiantes, docentes e instituciones superiores de estudio como son las universidades. Estos nuevos medios "posibilitan y urgen una nueva forma de organizar, representar y codificar la realidad". En consecuencia, es necesario 
desarrollar elementos de análisis crítico y de una formación del estudiantado que le permitan utilizar esa información de manera adecuada (Sevillano García, 2014, p. 297).

\section{Referencias}

Asociación Mexicana de Internet. (2015). $11^{\circ}$ estudio sobre los hábitos de los usuarios de internet en México 2015. Recuperado de https://amipci.org.mx/images/AMIPCI_HABITOS_DEL_INTERNAUTA_MEXICANO_2015.pdf el 26, 06, 2015

Centro de Estudios Especializados en Trastornos de Ansiedad. (2015). Hiperconectados: Adictos a la tecnología. Recuperado de http://www.ceeta.org/2015/06/14/hiperconectados-adictos-a-la-tecnología/ el 19, 09, 2015

Cope, B. y Kalantzis, M. (2009). Ubiquitous Learning. Exploring the anywhere/anytime possibilities for learning in the age of digital media. Estados Unidos: University of Illinois Press.

Diario ABC.es. (2015). La "nomofobia", o el miedo a salir de casa sin el "Smartphone", afecta ya al $75 \%$ de los españoles. $A B C$ TECNOLOGÍA. Recuperado de http://www.abc.es/tecnologia/noticias/20150804/abci-adictos-smartphone-jovenes201508041542html el 25, 08, 2015

Quicios, M. P. (2015). Los dispositivos digitales móviles en educación superior: Usos y experiencias. En E. Vázquez-Cano, \& M. L. Sevillano, (Edits.), Dispositivos digitales móviles en educación. El aprendizaje ubicuo. Madrid: Narcea.

Sevillano-García, M. L. (2014). Formación con información en la sociedad del conocimiento. En M. L. Sevillano García, Profanter Annemarie, M. C. Salgado Santamaría \& E. Vázquez Cano, Espacio europeo de educación, inicial, media, superior y permanente. Madrid: Ediciones Académicas.

Sevillano-García, M. L. (2015). El contexto socioeducativo de la ubicuidad y la movilidad. En E. Vázquez-Cano, \& M. L. Sevillano García (Edits), Dispositivos digitales móviles en educación. El aprendizaje ubicuo. Madrid: Narcea.

Tribuna. (13 de enero de 2012). Un modelo universitario para el siglo XXI. Diario El Mundo. 
Trillo, M. P. (2015). Principios pedagógicos del aprendizaje ubicuo. En E. Vázquez-Cano, \& M. L. Sevillano (Edits.), Dispositivos digitales móviles en educación. El aprendizaje ubicuo. Madrid: Narcea.

Vázquez-Cano, E. (2013). El videoartículo: Nuevo formato de divulgación en revistas científicas y su integración en MOOCs. Comunicar 41, 83- 91 DOI:http://dx.doi.org/10.3916/C41.2013.08

Vázquez-Cano, E, Sevillano García, M. L. \& Méndez Pérez, M. A. (2011). Programar en primaria y secundaria. Madrid: Pearson 